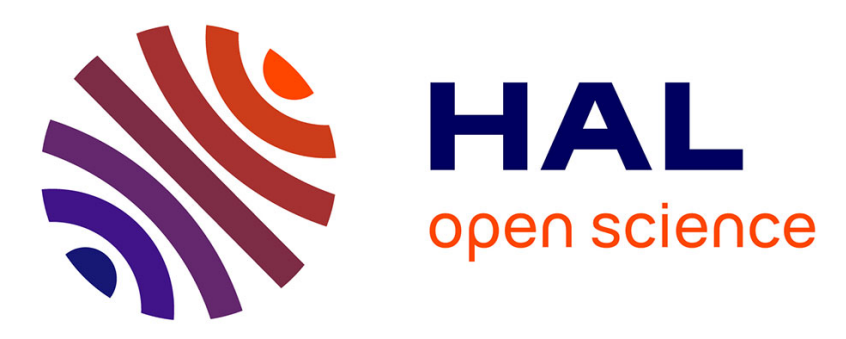

\title{
A dynamic high gain observer for a class of MIMO non triangular systems
}

Monia Oueder, Mondher Farza, Ridha Ben Abdennour, Mohammed M'Saad

\section{To cite this version:}

Monia Oueder, Mondher Farza, Ridha Ben Abdennour, Mohammed M'Saad. A dynamic high gain observer for a class of MIMO non triangular systems. The 18th IFAC World Congress on Automatic Control, Aug 2011, Milan, Italy. hal-01060170

\section{HAL Id: hal-01060170 https://hal.science/hal-01060170}

Submitted on 3 Sep 2014

HAL is a multi-disciplinary open access archive for the deposit and dissemination of scientific research documents, whether they are published or not. The documents may come from teaching and research institutions in France or abroad, or from public or private research centers.
L'archive ouverte pluridisciplinaire HAL, est destinée au dépôt et à la diffusion de documents scientifiques de niveau recherche, publiés ou non, émanant des établissements d'enseignement et de recherche français ou étrangers, des laboratoires publics ou privés. 


\title{
A dynamic high gain observer for a class of MIMO non triangular systems
}

\author{
M. Oueder ${ }^{*, * *}$ M. Farza ${ }^{* *}$ R. Ben Abdennour* M. M'Saad ${ }^{* *}$ \\ * Unité de Recherche CONPRI, Université de Gabès, ENIG, Tunisie \\ (oueder.monya@gmail.com, Ridha.benabdennour@enig.rnu.tn) \\ ** GREYC UMR 6072 CNRS, Université de Caen, ENSICAEN, France \\ (mondher.farza@unicaen.fr,mohammed.msaad@ensicaen.fr)
}

\begin{abstract}
A dynamic high gain like observer is proposed for a class of MIMO nonlinear systems that are observable for any inputs. Two main results are worth to be mentioned. The first one is related to the considered class of systems that is composed by cascade subsystems and each subsystem is associated to a subset of the outputs. Each subsystem assumes a triangular dependence on the state of that subsystem itself but may depend on the state of all other subsystems. Hence, the contribution is to extend the results existing in the literature in that more interconnections between the subsystems are allowed. The second important result lies in the nature of the observer gain which involves a scalar time-varying parameter governed by some scalar Riccati equation. Simulation results are given in order to highlight the performances of the proposed observer, namely its exponential convergence and a good behavior with respect to noise measurements.
\end{abstract}

Keywords: Nonlinear system. Dynamic high gain observer. Lyapunov Equation. Riccati equation. Exponentiel convergence.

\section{INTRODUCTION}

The need to study the observer design problem for nonlinear systems is, from a control point of view, well understood by now as pointed out by the contributions given by the list of references herein. Roughly speaking, the methods to design observers for nonlinear systems can be classified into four varieties. The first one which has met a great success in the past is based on the Kalman filter which is used as a nonlinear observer (Scharf and Sigurdsson [1984], Kalman and Bucy [1961]). The attractiveness of the Kalman filter is mainly due to its implementation simplicity independently of the system complexity. Nevertheless, a major drawback of this method still be the lack of guaranteed stability. The second approach is based on linearizable error dynamics where state transformations are exhibited in order to put the considered systems under a form where the nonlinearities depends only on the inputs and the outputs (Guay [2002], Hou and Pugh [1999], Krener and Isidori [1983], Souleiman et al. [2003], Xia and Gao [1989]). Thus, the resulting class of systems constitutes a subclass of triangular systems with the particularity that nonlinearities only depends on the measured inputs and outputs. The third approach consists in deeply exploring the LMI techniques, this leads to many studies over the last decade (Cho and Ro [2005], Tan and Edwards [2001]). More precisely, the gain of the observer is designed through the resolution of a LMI problem and as a consequence an observer exits only if the considered LMI problem is feasible (Fan and Arcak [2003], Rajamani [1998]). As pointed out in (Arcak and Kokotovic [2001]), the feasibility of the LMI problems considered is generally not known a priori and is to be determined numerically. The forth approach is based on the observable canonical form. The pioneer contribution has been made in (Gauthier et al. [1992]) where the authors gave a necessary and sufficient condition giving rise to the well known single output triangular canonical form. This canonical form is composed of a fixed linear dynamics component and a nonlinear triangular controlled one. Using this canonical form, the authors have designed a high gain observer under some global Lipschitz assumption on the controlled part. The gain of the proposed observer is issued from an algebraic Lyapunov equation that can be explicitly solved. Many generalizations of this result to systems with many outputs have been proposed (see e.g. Busawon et al. [1998], Hammouri and Farza [2003], Shim et al. [2001]). In (Shen et al. [2010]), a finite-time observer design for MIMO systems was also proposed. The main characteristic of the high gain observer proposed in (Gauthier et al. [1992]) lies in the easiness of its implementation since the observer is a copy of the model with a gain whose expression is explicitly given. Moreover the tuning of the observer is achieved through the choice of a single scalar design parameter. The effectiveness of such observer has been highlighted through many successful industrial applications (see e.g. (Gauthier et al. [1992], Farza et al. [1999])). There is however a main drawback of this observer that is worth to be noticed, namely the specification of the design parameter is generally carried out through a try and error procedure to get a satisfactory compromise between the accuracy of the observer and its sensitivity to noise measurements. Roughly speaking, the design parameter has to be chosen high enough with respect to the Lipschitz constant of the system nonlinearities. The aim of this paper is twofold. Firstly, one aims to extend the high gain observer design to a large class of systems that are observable for any input. The second main objective is to propose an alternative for the choice of the observer design parameter when designing a high gain observer. Indeed, the proposed observer is a high gain one and its gain is calibrated through a single parameter which is not constant, as in the usual high gain observers (Gauthier et al. [1992], Khalil and Saberi [1987]), but it is governed by a suitable scalar Riccati differential equation which takes advantage of the observer dynamics. As it shall be detailed later, the dynamics of the design parameter is such that this parameter continuously grows until some value and then decreases to a priori specified low value. At this low value, the observer still provide accurate estimates of the state while performing an appropriate insensitivity with respect noise measurements. The idea behind the use of a design parameter governed by a Riccati equation is not new and has been considered in other contributions dealing with output regulation (see e.g. Krishnamurthy and Khorrami [2004], Lei and Lin [2006], Praly [2003] and references therein). This paper is organized as follows. In the next section, the class of nonlinear systems which will be the subject of the observer design throughout this paper is introduced. Section 3 is dedicated to the recall of some preliminaries and the introduction of some variables that shall be used through the paper. In section 4 , the observer design is given and a full convergence analysis is provided. An academic example with simulation results is given in section 5 for illustration purposes.

\section{PROBLEM FORMULATION}

This paper exhibits a state observer for nonlinear systems which are diffeomorphic to:

$$
\left\{\begin{array}{l}
\dot{x}=A x+\varphi(u, x) \\
y=C x
\end{array}\right.
$$


where the state $x=\left(\begin{array}{llll}x^{1 T} & x^{2 T} \ldots x^{q T}\end{array}\right)^{T} \in R^{n}$, with $x^{k}=$ $\left(x_{1}^{k T} x_{2}^{k T} \ldots x_{\lambda_{k}}^{k T}\right)^{T} \in R^{n_{k}}, x_{i}^{k} \in R^{p_{k}}, i=1, \ldots, \lambda_{k}, k=1, \ldots, q$, $\sum_{k=1}^{q} n_{k}=\sum_{k=1}^{q} p_{k} \lambda_{k}=n$ with $p_{k} \geq 1$ and $\lambda_{k} \geq 2$; the output $y=\left(\begin{array}{llll}y_{1} & y_{2} & \ldots & y_{q}\end{array}\right)^{\mathrm{T}} \in R^{p}$ with $y_{k} \in R^{p_{k}}, k=1, \ldots, q$ and $\sum_{k=1}^{q} p_{k}=p$;

$$
\begin{gathered}
A=\left[\begin{array}{ccc}
A_{1} & & \\
& \ddots & \\
& & A_{q}
\end{array}\right], A_{k}=\left[\begin{array}{cccc}
0 & I_{p_{k}} & & 0 \\
\vdots & & \ddots & \\
0 & \ldots & 0 & I_{p_{k}} \\
0 & \ldots & 0 & 0
\end{array}\right] \\
C=\operatorname{diag}\left(C_{1} \ldots C_{q}\right), C_{k}=\left[\begin{array}{llll}
I_{p_{k}} & 0 & \ldots & 0
\end{array}\right]
\end{gathered}
$$

and the nonlinear function field $\varphi(u, x)=\left(\varphi^{1 T}(u, x), \ldots, \varphi^{q T}(u, x)\right)^{T} \in$ $R^{n} ; \varphi^{k}(u, x)=\left(\varphi_{1}^{k T}(u, x), \ldots, \varphi_{\lambda_{k}}^{k T}(u, x)\right)^{T} \in R^{n_{k}}$ where for $k=$ $1, \ldots, q$, the function $\varphi_{i}^{k}(u, x) \in R^{p_{k}}$ is differentiable with respect to $x$ and assumes the following structural dependence on the states:

- for $1 \leq i \leq \lambda_{k}-1$ :

$$
\varphi_{i}^{k}(u, x)=\varphi_{i}^{k}\left(u, x^{1}, \ldots, x^{k-1}, x_{1}^{k}, \ldots, x_{i}^{k}, x_{1}^{k+1}, \ldots, x_{1}^{q}\right)
$$

- for $i=\lambda_{k}$ :

$$
\varphi_{\lambda_{k}}^{k}(u, x)=\varphi_{\lambda_{k}}^{k}\left(u, x^{1}, x^{2}, \ldots, x^{q}\right)
$$

Our objective is to whose gain is derived from an algebraic Lyapunov equation and which is calibrated by the choice of a single design parameter. However, unlike the classical high gain observer (Gauthier et al. [1992]) and in order to improve the performance of such observer with respect to noise rejection, the design parameter has not to be chosen constant but time-varying with a dynamics which allows it to be maintained at low values while the observer continues to provide accurate estimates.

\section{PRELIMINARIES}

In this section, one shall introduce some variables used in the observer equations. A technical lemma needed in the proof of the convergence of the observation error, is also given.

3.1 Some definitions and notations

Let $\theta: R \rightarrow R, t \mapsto \theta(t)$ be a real-valued function and for $k=1, \ldots, q$, let $\Delta_{k}(\theta)$ be the diagonal matrix defined by:

$$
\Delta_{k}(\theta)=\operatorname{diag}\left(\frac{1}{\theta^{\delta_{k}}} I_{p_{k}}, \frac{1}{\theta^{2 \delta_{k}}} I_{p_{k}}, \ldots, \frac{1}{\theta^{\lambda_{k} \delta_{k}}} I_{p_{k}}\right)
$$

and one defines $\delta_{k}$ which indicates the power of $\theta$ as follows:

$$
\delta_{k}=2^{q-k}\left(\prod_{i=k+1}^{q} \lambda_{i}-1\right), \text { for } k=1, \ldots, q-1 ; \delta_{q}=1
$$

Notice that for any $k=1, \ldots, q-1$, one has

$$
\frac{\delta_{k}}{2}=\left(\lambda_{k+1}-1\right) \delta_{k+1}
$$

Since $\lambda_{k} \geq 2$, the $\delta_{q}$ 's constitute a non increasing sequence of positive real numbers, i.e.

$$
\delta_{1} \geq \delta_{2} \geq \ldots \geq \delta_{q}=1
$$

One now shall give a lemma needed in the proof of the observation error convergence. This lemma allows to provide a sequence of reals that reflects in some sense the interconnections between the blocks nonlinearities. Its proof is similar to that given in (Liu et al. [2008]).

Lemma 3.1. For $k, l=1, \ldots, q$ and $i=1, \ldots, \lambda_{k}, j=2, \ldots, \lambda_{l}-$ 1, one defines $\chi_{l, j}^{k, i}$ as follows: $\chi_{l, j}^{k, i}=0$ if $\frac{\partial \varphi_{i}^{k}}{\partial x_{j}^{l}} \equiv 0 ; \chi_{l, j}^{k, i}=$ 1 otherwise. Now, consider the following sequence of reals:

$$
\begin{aligned}
& \sigma_{i}^{k}=\sigma_{1}^{k}+i \delta_{k}, k=1, \ldots, q \text { and } i=1, \ldots, \lambda_{k} \\
& \text { where } \sigma_{1}^{k}=-\lambda_{k} \delta_{k}+\lambda_{1} \delta_{1}+1
\end{aligned}
$$

and the $\delta_{k}$ 's are given by (7). Then, the terms of this sequence satisfy the following two properties:

$$
\begin{aligned}
& (P 1) \quad 1=\sigma_{1}^{1}>\sigma_{1}^{2}>\ldots>\sigma_{1}^{q} \\
& (P 2) \text { if } \chi_{l, j}^{k, i}=1 \text { then } \sigma_{j}^{l}-\sigma_{i}^{k}-\frac{\delta_{k}}{2}-\frac{\delta_{l}}{2} \leq-\frac{1}{2}
\end{aligned}
$$

Notice that the reals $\sigma_{1}^{k}$ given by Lemma 3.1. also satisfy the following properties

$$
\sigma_{\lambda_{k}}^{k} \triangleq \sigma_{1}^{k}+\lambda_{k} \delta_{k}=1+\lambda_{1} \delta_{1}=\sigma_{\lambda_{1}}^{1} \text { for } k=1, \ldots, q
$$

Before ending this section, one shall define some matrices that shall be used throughout this paper. Indeed, for $k=1, \ldots, q$, set

$$
\Lambda_{k}(\theta)=\theta^{-\sigma_{1}^{k}} \Delta_{k}(\theta)
$$

where the $\sigma_{1}^{k}$,s are the non negative reals given by Lemma 3.1. and $\Delta_{k}(\theta)$ is defined by (6). Taking into account the structure of $A_{k}$ and $C_{k}$ respectively given by (2) and (3), one can show that the following identities hold:

$$
\Lambda_{k}(\theta) A_{k} \Lambda_{k}^{-1}(\theta)=\theta^{\delta_{k}} A_{k}, C_{k} \Lambda_{k}^{-1}(\theta)=\theta^{\sigma_{1}^{k}+\delta_{k}} C_{k}
$$

Moreover, one can check that

$$
\dot{\Lambda}_{k}(\theta) \triangleq \frac{d}{d t} \Lambda_{k}(\theta)=-\frac{\dot{\theta}(t)}{\theta(t)} \Lambda_{k}(\theta)\left(\sigma_{1}^{k} I_{n_{k}}+\delta_{k} D_{k}\right)
$$

where $I_{n_{k}}$ is the $n_{k} \times n_{k}$ identity matrix and $D_{k}$ is the following $n_{k} \times n_{k}$ diagonal matrix $D_{k}=\operatorname{diag}\left(I_{p_{k}}, 2 I_{p_{k}}, \ldots, \lambda_{k} I_{p_{k}}\right)$. Now, for $k=1, \ldots, q$, let

$$
K_{k}^{T}=\left[\begin{array}{llll}
K_{k, 1}^{T} & K_{k, 2}^{T} & \cdots & K_{k, \lambda_{k}}^{T}
\end{array}\right]^{T}
$$

be the $n_{k} \times p_{k}$ matrix, with $K_{k, i} \in R^{p_{k} \times p_{k}}$, such that $\tilde{A}_{k} \triangleq A_{k}-$ $K_{k} C_{k}$ is Hurwitz. Then, there exist a strictly positive real number $a>0$ and $q$ symmetric positive definite matrices $S_{k}, n_{k} \times n_{k}$ (Krishnamurthy and Khorrami. [2002], Lei and Lin [2006]) such that

$$
\tilde{A}_{k}^{T} S_{k}+S_{k} \tilde{A}_{k} \leq-a S_{k} \text { and } D_{k} S_{k}+S_{k} D_{k} \geq 0
$$

Set

$$
\begin{aligned}
& Q_{k}=S_{k} D_{k}+D_{k} S_{k} ; \quad \Omega_{k}=\delta_{k} Q_{k}+2 \sigma_{1}^{k} S_{k} \\
& \Omega=\operatorname{diag}\left(\Omega_{1}, \Omega_{2}, \ldots, \Omega_{q}\right) ; \quad S=\operatorname{diag}\left(S_{1}, S_{2}, \ldots, S_{q}\right)
\end{aligned}
$$

It is easy to see that since $Q_{k} \geq 0$ (according to (18)), $\Omega_{k}$ is symmetric positive definite and so is $\Omega$. In the sequel, one shall denote by $\lambda_{M}(\cdot)$ and $\lambda_{m}(\cdot)$ the largest and the smallest eigenvalue of $(\cdot)$, respectively. The conditioning number of $(\cdot)$ shall be denoted by $\mu(\cdot)$, i.e. $\mu(\cdot)=\frac{\lambda_{M}(\cdot)}{\lambda_{m}(\cdot)}$. 4. OBSERVER DESIGN

As generally assumed in the high gain observer design (Gauthier et al. [1992], Farza et al. [2004], Shen and Xia. [2008]), one considers the following Lipschitz assumption:

Assumption 1. $\varphi(u, x)$ is a globally Lipschitz nonlinear function with respect to $x$ uniformly in $u$.

\subsection{Dynamic high gain observer}

The candidate observer borrows from the usual high gain observer up to appropriate change in the involved design as pointed out by the following equations

$$
\left\{\begin{array}{l}
\text { for } k=1, \ldots, q: \\
\dot{\hat{x}}^{k}(t)=A_{k} \hat{x}^{k}(t)+\varphi^{k}(u(t), \underline{\hat{x}}(t))-\Delta_{k}^{-1}(\theta) K_{k} C_{k} e^{k}(t) \\
\dot{\theta}(t)=-\frac{\mu_{1}}{2} \theta(t)(a(\theta(t)-1)-g(t) \gamma(\|\tilde{y}(t)\|)) \\
\quad \text { with } \theta(0) \geq 1 \\
g(t)=\frac{M}{1+\min \left(\rho, \frac{1}{T} \int_{\max (0, t-T)}^{t}\|\tilde{y}(\tau)\|^{2} d \tau\right)}
\end{array}\right.
$$

with

- $\hat{x}=\left(\begin{array}{llll}\hat{x}^{1 T} & \hat{x}^{2 T} & \ldots & \hat{x}^{q T}\end{array}\right)^{T} \in R^{n}$, $\hat{x}^{k}=\left(\begin{array}{llll}\hat{x}_{1}^{k T} & \hat{x}_{2}^{k T} & \ldots & \hat{x}_{\lambda_{k}}^{k T}\end{array}\right)^{T} \in R^{n_{k}}, \hat{x}_{i}^{k} \in R^{p_{k}}, i=1, \ldots, \lambda_{k}$, $k=1, \ldots, q, \sum_{k=1}^{q} n_{k}=n$.

- $\underline{\hat{x}}_{1}^{k}=x_{1}^{k}$ (output injection) for $k=1, \ldots, q$.

- $\underline{\hat{\hat{x}}}_{i}^{k}=\hat{x}_{i}^{k}$ if $i \neq 1$.

- $e^{k}=\hat{x}^{k}-x^{k}, \quad \tilde{y}=C e=C(\hat{x}-x), \quad \mu_{1}=\frac{\lambda_{m}(S)}{\lambda_{M}(\Omega)}$.

- $u$ and $y$ are known inputs and outputs of system (1).

- The reals $\rho, T$ and $M$ are positive and are the observer design parameters.

- $\gamma: R \longrightarrow R^{+},\|\tilde{y}\| \mapsto \gamma(\|\tilde{y}\|)$ is a real-valued non negative, non decreasing and bounded function satisfying $\gamma(0)=0$. 
One now states the main result of this paper.

Theorem 4.1. Under Assumption 1., the trajectories of system (20) converge exponentially to those of system (1) for relatively high values of the parameter $M$.

The proof of the theorem is given below.

\subsection{Convergence Analysis}

First of all, one shall prove the boundedness of $\theta(t)$ and give expressions of the corresponding lower and upper bounds. To this end, one shall consider two cases depending on the sign of $\dot{\theta}(t)$. Before considering these cases, one notices that the expression of $\dot{\theta}$ in (20) is such that: $\theta=1 \Longrightarrow \dot{\theta} \geq 0$. As a result, one has $\theta(t) \geq 1$ for all $t \geq 0$ as soon as $\theta(0) \geq 1$. One also notices that: $\forall t \geq 0: 0<g(t) \leq M$. Let us now discuss both cases mentioned above.

- $\dot{\theta}(t) \geq 0$ : Since $\theta(t) \geq 1$ and according to (20), one has $a(\theta(t)-1)-g(t) \gamma(\|\tilde{y}\|) \leq 0$ which implies that $\theta(t) \leq 1+\frac{M}{a} \gamma(\|\tilde{y}\|)$.

$$
\text { Thus, } \quad \forall t \geq 0: 1 \leq \theta(t) \leq 1+\frac{M \gamma_{\max }}{a}
$$

where $\gamma_{\max }$ is the upper bound of $\gamma$.

- $\dot{\theta}(t) \leq 0$ : Since $\theta(t) \geq 1$ and from (20), one has:

$$
\dot{\theta}(t) \leq-\frac{a \mu_{1}}{2} \theta(t)+\frac{a \mu_{1}}{2}\left(1+\frac{M \gamma_{\max }}{a}\right)
$$

Integrating (22) from some $t_{0}<t$ to $t$ gives

$$
\theta(t) \leq \theta\left(t_{0}\right)+\left(1+\frac{M \gamma_{\max }}{a}\right)
$$

Now, the time $t_{0}$ may be either 0 , in which case $\theta(0)$ is arbitrary, either the final time of an interval on which $\theta(t) \geq 0$ and according to $(21)$, one has $\theta\left(t_{0}\right) \leq 1+\frac{M \gamma_{\max }}{a}$. As a result, for any $t_{0}$, one can choose $M$ high enough such that $\theta\left(t_{0}\right) \leq 1+\frac{M \gamma_{\max }}{a}$ and (23) becomes

$$
\theta(t) \leq 2\left(1+\frac{M \gamma_{\max }}{a}\right)
$$

To summarize and according to (21) and (24), $\theta(t)$ is bounded and satisfies:

$$
\forall t \geq 0: \theta(t) \leq \theta_{\max } \triangleq 2\left(1+\frac{M \gamma_{\max }}{a}\right)
$$

Let us, now, derive the time derivative of $e(t)=\hat{x}(t)-x(t)$ and let $e^{k}(t)$ be the $k^{\prime} t h$ subcomponent of $e(t)$. For writing convenience and as long as there is no ambiguity, one shall omit the time $t$ for each variable. One has:

$$
\dot{e}^{k}=A_{k} e^{k}+\varphi^{k}(u, \underline{\hat{x}})-\varphi^{k}(u, x)-\Delta_{k}^{-1}(\theta) K_{k} C_{k} e^{k}
$$

where $u$ is an admissible control such that $\|u\|_{\infty} \leq \eta, \eta>0$ is a given constant. For $k=1, \cdots, q$, set

$$
\bar{e}^{k}=\Lambda_{k}(\theta) e^{k}
$$

From equation (26) and using (15) and (16), one gets:

$$
\dot{\bar{e}}^{k}=\theta^{\delta_{k}} \tilde{A}_{k} \bar{e}^{k}+\Lambda_{k}(\theta)\left(\varphi^{k}(u, \underline{\hat{x}})-\varphi^{k}(u, x)\right)-\frac{\dot{\theta}}{\theta}\left(\sigma_{1}^{k} I_{k}+\delta_{k} D_{k}\right) \bar{e}^{k}
$$

Set

$$
V_{k}\left(\bar{e}^{k}\right)=\bar{e}^{k^{T}} S_{k} \bar{e}^{k}
$$

and let $V(\bar{e})=\sum_{k=1}^{q} V_{k}\left(\bar{e}^{k}\right)=\bar{e}^{T} S \bar{e}$ where $S$ is given by (19), be the candidate Lyapunov function. Please notice that according to (27) and (28), one has $\forall t \geq 0:\left\|e^{k}(t)\right\|^{2} \leq\left\|\bar{e}^{k}(t)\right\|^{2} \leq \frac{1}{\lambda_{m}\left(S_{k}\right)} V_{k}\left(\bar{e}^{k}(t)\right.$ and as a result one has

$$
\forall t \geq 0:\|e(t)\| \leq\|\bar{e}(t)\| \leq \frac{1}{\sqrt{\lambda}_{m}(S)} \sqrt{V(\bar{e}(t))}
$$

Using (18), one can show that

$$
\begin{aligned}
\dot{V}_{k} & \leq-a \theta^{\delta_{k}} V_{k}-\frac{\dot{\theta}}{\theta} \bar{e}^{k^{T}} \Omega_{k} \bar{e}^{k} \\
& +2 \sqrt{\lambda_{M}(S)} \sqrt{V_{k}} \sum_{i=1}^{\lambda_{k}} \frac{1}{\theta^{\sigma_{i}^{k}}}\left\|\left(\varphi_{i}^{k}(u, \underline{\hat{x}})-\varphi_{i}^{k}(u, x)\right)\right\|
\end{aligned}
$$

where $\Omega_{k}$ is given by (19), $\sigma_{i}^{k}$ and $\sigma_{j}^{l}$ are as given in (10). Then,

$$
\begin{aligned}
\dot{V}_{k} \leq & -a \theta^{\delta_{k}} V_{k}-\frac{\dot{\theta}}{\theta} \bar{e}^{k^{T}} \Omega_{k} \bar{e}^{k} \\
& +2 \rho_{k} \sqrt{\lambda_{M}(S)} \sqrt{V_{k}} \sum_{i=1}^{\lambda_{k}} \sum_{l=1}^{q} \sum_{j=2}^{\lambda_{l}} \chi_{l, j}^{k, i} \theta^{-\sigma_{i}^{k}}\left\|e_{j}^{l}\right\|
\end{aligned}
$$

where $\rho_{k}=\sup \left\{\left\|\frac{\partial \varphi_{i}^{k}}{\partial x_{j}^{l}}(u, x)\right\| ; x \in R^{n}\right.$ and $\left.\|u\|_{\infty} \leq \eta\right\}$ and the $\chi_{l, j}^{k, i}$, s have the same definition as in Lemma 3.1.. Hence

$$
\begin{aligned}
\dot{V}_{k} \leq & -a \theta^{\delta_{k}} V_{k}-\frac{\dot{\theta}}{\theta} \bar{e}^{k^{T}} \Omega_{k} \bar{e}^{k} \\
& +2 \rho_{k} \sqrt{\lambda_{M}(S)} \sqrt{V_{k}} \sum_{i=1}^{\lambda_{k}} \sum_{l=1}^{q} \sum_{j=2}^{\lambda_{l}} \chi_{l, j}^{k, i} \theta^{\sigma_{j}^{l}-\sigma_{i}^{k}}\left\|\bar{e}_{j}^{l}\right\|
\end{aligned}
$$

where $\sigma_{j}^{l}$ is defined as in (10), i.e. $\sigma_{j}^{l}=\sigma_{1}^{l}+j \delta_{l}$. This leads to

$$
\begin{aligned}
& \dot{V}_{k} \leq-a \theta^{\delta_{k}} V_{k}+2 \rho_{k} \mu(S) \sqrt{\theta^{\delta_{k} V_{k}}}
\end{aligned}
$$

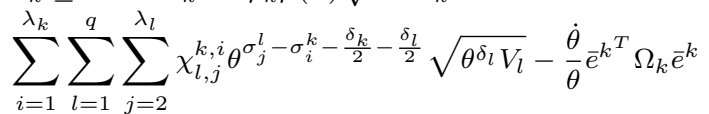

Now, since $\theta(t) \geq 1$ and according to Lemma 3.1., one has

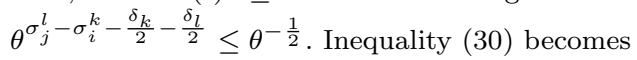

$$
\begin{aligned}
& \dot{V}_{k} \leq-a \theta^{\delta_{k}} V_{k}-\frac{\dot{\theta}}{\theta} \bar{e}^{k^{T}} \Omega_{k} \bar{e}^{k}+2 \lambda_{k} \rho_{k} \mu(S) \theta^{-\frac{1}{2}} \sqrt{\theta^{\delta_{k} V_{k}}} \times \\
& \sum_{l=1}^{q} \sum_{j=2}^{\lambda_{l}} \sqrt{\theta^{\delta_{l} V_{l}}}
\end{aligned}
$$

Set $W_{k}\left(\bar{e}^{k}\right)=\bar{e}^{k^{T}} \Omega_{k} \bar{e}^{k}$ and $W(\bar{e})=\sum_{k=1}^{q} W_{k}\left(\bar{e}^{k}\right)$. One has $\mu_{1} W_{k} \triangleq \frac{\lambda_{m}(S)}{\lambda_{M}(\Omega)} W_{k} \leq V_{k} \leq \frac{\lambda_{M}(S)}{\lambda_{m}(\Omega)} W_{k} \triangleq \mu_{2} W_{k}$. One recalls that $\frac{\mu_{2}}{\mu_{1}}=\mu(S) \mu(\Omega)$. So, inequality $(31)$ becomes

$$
\dot{V}_{k} \leq-a \theta^{\delta_{k}} V_{k}-\frac{\dot{\theta}}{\theta} W_{k}+2 \lambda_{k} \rho_{k} \mu(S) \theta^{-\frac{1}{2}} \sqrt{\theta^{\delta_{k}} V_{k}} \sum_{l=1}^{q} \sum_{j=2}^{\lambda_{l}} \sqrt{\theta^{\delta_{l}} V_{l}}
$$

Now, for $k=1, \cdots, q$, set $V_{k}^{*}=a \theta^{\delta_{k}} V_{k}$ and let $V^{*}=\sum_{k=1}^{q} V_{k}^{*}$. Notice that since $\theta \geq 1$ and according to (9), one has $a \theta V=a \theta^{\delta_{q}} V \leq$ $V^{*} \leq a \theta^{\delta_{1}} V$. Then,

$$
\begin{aligned}
\dot{V}_{k} & \leq-V_{k}^{*}+2 \lambda_{k} \frac{\rho_{k}}{a} \mu(S) \theta^{-\frac{1}{2}} \sqrt{V_{k}^{*}} \sum_{l=1}^{q} \sum_{j=2}^{\lambda_{l}} \sqrt{V_{l}^{*}}-\frac{\dot{\theta}}{\theta} W_{k} \\
& \leq-V_{k}^{*}+2 \lambda_{k} n \frac{\rho_{k}}{a} \mu(S) \theta^{-\frac{1}{2}} V^{*}-\frac{\dot{\theta}}{\theta} W_{k}
\end{aligned}
$$

Hence, $\dot{V} \leq-V^{*}+2 n^{2} \frac{\rho}{a} \mu(S) \theta^{-\frac{1}{2}} V^{*}-\frac{\dot{\theta}}{\theta} W$, where $\rho=\max \left\{\rho_{k}, 1 \leq\right.$ $k \leq q\}$. Substituting $\frac{\dot{\theta}}{\theta}$ by its expression, one gets

$$
\dot{V} \leq-V^{*}+2 n^{2} \frac{\rho}{a} \mu(S) \theta^{-\frac{1}{2}} V^{*}+\frac{\mu_{1}}{2}(a(\theta-1)-g(t) \gamma(\|\tilde{y}\|)) W
$$

Two cases shall be considered depending on whether $\theta(t)$, governed by the differential equation in (20), is greater or not than

$$
\theta_{c} \triangleq 4^{2}\left(2 n^{2} \frac{\rho}{a} \mu(S)\right)^{2}
$$

- Case 1: $\theta(t)>\theta_{c}$. One can check that

$$
\theta(t)-4^{2}\left(2 n^{2} \frac{\rho}{a} \mu(S)\right)^{2}>0 \Longrightarrow 1-\left(2 n^{2} \frac{\rho}{a} \mu(S)\right) \theta^{-\frac{1}{2}}(t)>\frac{3}{4}
$$

Using (32) and (34), inequality (32) becomes:

$$
\begin{aligned}
\dot{V}(t) & \leq-\left(1-2 n^{2} \frac{\rho}{a} \mu(S) \theta^{-\frac{1}{2}}\right) V^{*}+\frac{\mu_{1}}{2}(a(\theta-1)-g(t) \gamma(\|\tilde{y}\|)) W \\
& \leq-\frac{1}{4} a(\theta+1) V(t)-\frac{\mu_{1}}{4 \mu_{2}} g(t) \gamma(\|\tilde{y}\|) V(t)
\end{aligned}
$$

- Case $2: \theta(t) \leq \theta_{c}$. Using (32) and the fact that $\theta(t) \geq 1$, inequality (32) becomes: 


$$
\begin{aligned}
\dot{V} & \leq-V^{*}+\frac{\theta_{c}^{\frac{1}{2}}}{4} \theta^{-\frac{1}{2}} V^{*}+\frac{\mu_{1}}{2}(a(\theta-1)-g(t) \gamma(\|\tilde{y}\|)) W \\
& \leq-\frac{1}{4} a(\theta+1) V(t)+\frac{a \theta_{c}^{\delta_{1}+\frac{1}{2}}}{4} V-\frac{\mu_{1}}{4 \mu_{2}} g(t) \gamma(\|\tilde{y}\|) V
\end{aligned}
$$

Comparing (35) and (35), one can easily conclude that for any $\theta$, one has:

$$
\dot{V} \leq-\frac{a}{4}(\theta+1) V+\frac{a \theta_{c}^{\delta_{1}+\frac{1}{2}}}{4} V-\frac{\mu_{1}}{4 \mu_{2}} g(t) \gamma(\|\tilde{y}\|) V
$$

The remaining of the proof is as follows. One shall firstly show that $\|\tilde{y}(t)\|$ is bounded. Then, One shall prove the boundedness of $\|e(t)\|$. Finally, the convergence to zero of $\|\tilde{y}(t)\|$ and $\|e(t)\|$ will be established. Let us show that $\|\tilde{y}(t)\|$ is bounded. Indeed, this shall be done by contradiction. Suppose the contrary and choose $\rho$ high enough. Then,

$$
\begin{aligned}
& \exists K>0 ; \exists t^{\star}>T ; \forall t \geq t^{\star}-T: \\
& \|\tilde{y}(t)\|>K \text { and } \frac{1}{T} \int_{t^{\star}-T}^{t}\|\tilde{y}(\tau)\|^{2} d \tau>\rho
\end{aligned}
$$

As a result, one has $\forall t \geq t^{\star}-T:\|\tilde{y}(t)\|>K$ and $g(t)=\frac{M}{1+\rho}$. Let $\gamma_{K}=\gamma(K)$. Since $\gamma$ is a positive non decreasing function of $\|\tilde{y}\|$, one has: $\forall t \geq t^{\star}-T: \gamma(\|\tilde{y}(t)\|) \geq \gamma_{K}$. Let us choose $M$ as follows

$$
M=\frac{\lambda(1+\rho)}{\gamma_{K}}
$$

where $\lambda$ is a positive constant and is such that $\lambda>\theta_{c}^{\delta_{1}+\frac{1}{2}} \frac{\mu_{2}}{\mu_{1}}$. For $t \geq t^{\star}-T$, using (37), inequality (36) becomes:

$\dot{V}(\bar{e}(t)) \leq-\frac{1}{4} a(\theta+1) V(\bar{e}(t))+\frac{a \theta_{c}^{\delta_{1}+\frac{1}{2}}}{4} V(\bar{e}(t))-\frac{\mu_{1}}{4 \mu_{2}} \frac{M \gamma_{K}}{1+\rho} V(\bar{e}(t))$ Substituting $M$ by its expression, (38), one gets

$$
\dot{V}(\bar{e}(t)) \leq-\left(\frac{\mu_{1}}{\mu_{2}} \lambda-\theta_{c}^{\delta_{1}+\frac{1}{2}}\right) \frac{1}{4} V(\bar{e}(t))
$$

Thus, for all $t \geq t^{\star}-T$, one has

$$
V(\bar{e}(t)) \leq \exp \left(-\left(\frac{\mu_{1}}{\mu_{2}} \lambda-\theta_{c}^{\delta_{1}+\frac{1}{2}}\right) t\right) V\left(\bar{e}\left(t^{\star}-T\right)\right)
$$

Since $V\left(\bar{e}\left(t^{\star}-T\right)\right)$ is constant, one can choose $\lambda$ such that

$$
\exp \left(-\left(\frac{\mu_{1}}{\mu_{2}} \lambda-\theta_{c}^{\delta_{1}+\frac{1}{2}}\right) t\right) V\left(\bar{e}\left(t^{\star}-T\right)\right)<\lambda_{m}(S) \frac{K^{2}}{4}
$$

Combining (40), (41) and (29), one gets for $t \geq t^{\star}-T$ : $\|\tilde{y}(t)\|^{2} \leq$ $\|e(t)\|^{2} \leq\|\bar{e}(t)\|^{2} \leq \frac{1}{\lambda_{m}(S)} V(\bar{e}(t))<\frac{K^{2}}{4}$, i.e. $\|\tilde{y}(t)\|<\frac{K}{2}$. This is in contradiction with $(37)$ and $\|\tilde{y}(t)\|$ is then bounded. In the sequel, one shall denote by $B_{\tilde{y}}$ the upper bound of $\|\tilde{y}(t)\|$, i.e.

$$
\forall t \geq 0:\|\tilde{y}(t)\| \leq B_{\tilde{y}}
$$

Let us now show the boundedness of $\|e(t)\|$. To this end, we introduce the following change of coordinates

$$
\tilde{e}^{k}=\Lambda_{k}(\bar{\theta}) e^{k}, k=1, \ldots, q
$$

where $\bar{\theta}$ is a constant satisfying

$$
\bar{\theta} \geq \max \left\{\theta_{\max }, \theta_{c}\right\}
$$

where $\theta_{\max }$ and $\theta_{c}$ are respectively given by (25) and (33). Then the error dynamics (26) is transformed into

$$
\begin{aligned}
& \dot{e}^{k}=A_{k} e^{k}-\Delta_{k}^{-1}(\bar{\theta}) K_{k} C_{k} e^{k}+\varphi^{k}(u, \underline{\hat{x}})-\varphi^{k}(u, x) \\
& +\Delta_{k}^{-1}(\bar{\theta}) K_{k} C_{k} e^{k}-\Delta_{k}^{-1}(\theta) K_{k} C_{k} e^{k}
\end{aligned}
$$

Using (43) and (45) and proceeding as above, one gets:

$$
\begin{aligned}
\dot{\tilde{e}}^{k}= & \bar{\theta}^{\delta_{k}}\left(A_{k}-K_{k} C_{k}\right) \tilde{e}^{k}+\Lambda(\bar{\theta})\left(\varphi^{k}(u, \underline{\hat{x}})-\varphi^{k}(u, x)\right)+\bar{\theta}^{-\sigma_{1}^{k}} K_{k} \tilde{y}_{k} \\
& -\bar{\theta}^{-\sigma_{1}^{k}} \Delta^{-1}\left(\frac{\theta}{\bar{\theta}}\right) K_{k} \tilde{y}_{k}
\end{aligned}
$$

Now set $\tilde{V}_{k}\left(\tilde{e}^{k}\right)=\tilde{e}^{k T} S_{k} \tilde{e}^{k}$ and consider the Lyapunov function $\tilde{V}(\tilde{e})=\sum_{k=1}^{q} \tilde{V}_{k}\left(\tilde{e}^{k}\right)$ for system (45). Again, proceeding as above, one can show that (see inequality (31)):

$$
\begin{array}{r}
\dot{\tilde{V}}_{k} \leq-a \bar{\theta}^{\delta_{k}} \tilde{V}_{k}+2 \lambda_{k} \rho_{k} \mu(S) \bar{\theta}^{-\frac{1}{2}} \sqrt{\bar{\theta}^{\delta_{k}} \tilde{V}_{k}} \sum_{l=1}^{q} \sum_{j=1}^{\lambda_{l}} \sqrt{\bar{\theta}^{\delta_{l}} \tilde{V}_{l}} \\
+\bar{\theta}^{-\sigma_{1}^{k}} \tilde{e}^{k T} S_{k} K_{k} \tilde{y}_{k}-\bar{\theta}^{-\sigma_{1}^{k}} \tilde{e}^{k T} S_{k} \Delta_{k}^{-1}\left(\frac{\theta}{\bar{\theta}}\right) K_{k} \tilde{y}_{k}
\end{array}
$$

Now, for $k=1, \cdots, q$, set $\tilde{V}_{k}^{*}=\bar{\theta}^{\delta_{k}} \tilde{V}_{k}$ and let $\tilde{V}^{*}=\sum_{k=1}^{q} \tilde{V}_{k}^{*}$. Notice that

$$
\bar{\theta} \tilde{V} \leq \tilde{V}^{\star} \leq \bar{\theta}^{\delta_{1}} \tilde{V}
$$

Then

$$
\begin{aligned}
\dot{\tilde{V}}_{k} \leq & -a \tilde{V}_{k}^{*}+2 \lambda_{k} n \rho_{k} \mu(S) \bar{\theta}^{-\frac{1}{2}} \tilde{V}^{*}+\bar{\theta}^{-\sigma_{1}^{k}} \tilde{e}^{k T} S_{k} K_{k} \tilde{y}_{k} \\
& -\bar{\theta}^{-\sigma_{1}^{k}} \tilde{e}^{k T} S_{k} \Delta_{k}^{-1}\left(\frac{\theta}{\bar{\theta}}\right) K_{k} \tilde{y}_{k}
\end{aligned}
$$

Hence

$$
\begin{aligned}
\dot{\tilde{V} \leq} & -a \tilde{V}^{*}+2 n^{2} \rho \mu(S) \bar{\theta}^{-\frac{1}{2}} \tilde{V}^{*} \\
& +\sum_{k=1}^{q}\left(\bar{\theta}^{-\sigma_{1}^{k}} \tilde{e}^{k T} S_{k} K_{k} \tilde{y}_{k}-\bar{\theta}^{-\sigma_{1}^{k}} \tilde{e}^{k T} S_{k} \Delta_{k}^{-1}\left(\frac{\theta}{\bar{\theta}}\right) K_{k} \tilde{y}_{k}\right)
\end{aligned}
$$

According to the choice of $\bar{\theta},\left(1-2 n^{2} \rho \mu_{S} \bar{\theta}^{-\frac{3}{4}}\right)>\frac{1}{2}$. Using (46), one gets

$\dot{\tilde{V}} \leq-a \frac{3}{4} \bar{\theta} \tilde{V}+\sum_{k=1}^{q}\left(\bar{\theta}^{-\sigma_{1}^{k}} \tilde{e}^{k T} S_{k} K_{k} \tilde{y}_{k}-\bar{\theta}^{-\sigma_{1}^{k}} \tilde{e}^{k T} S_{k} \Delta_{k}^{-1}\left(\frac{\theta}{\bar{\theta}}\right) K_{k} \tilde{y}_{k}\right)$

Now, it is clear that

$$
\begin{array}{r}
\sum_{k=1}^{q} \bar{\theta}^{-\sigma_{1}^{k}} \tilde{e}^{k} S_{k} K_{k} \tilde{y}_{k} \leq \bar{\theta}^{-\sigma_{1}^{k}} \frac{\left\|K_{k}\right\|}{\lambda_{m}(S)} \sqrt{\tilde{V}} B_{\tilde{y}} \\
\sum_{k=1}^{q} \bar{\theta}^{-\sigma_{1}^{k}} \tilde{e}^{k T} S_{k} \Delta_{k}^{-1}\left(\frac{\theta}{\bar{\theta}}\right) K_{k} \tilde{y}_{k} \leq \bar{\theta}^{-\sigma_{1}^{k}}\left\|K_{k}\right\| \mu(S) \sqrt{\tilde{V}} B_{\tilde{y}}
\end{array}
$$

where $B_{\tilde{y}}$ is the upper bound of $\tilde{y}(t)$ as given in (42). In view of the estimations above, we have

$$
\dot{\tilde{V}} \leq-\frac{3}{4} a \bar{\theta} \tilde{V}+\left(K_{M} \bar{\theta}^{-\sigma_{1}^{k}}\left(\mu(S)+\frac{1}{\lambda_{m}(S)}\right) B_{\tilde{y}}\right) \sqrt{\tilde{V}}
$$

where $K_{M}=\max _{1 \leq k \leq q}\left\|K_{k}\right\|$. This yields to

$$
\sqrt{\tilde{V}(\tilde{e}(t))} \leq \exp \left(-\frac{3}{8} a \bar{\theta} t\right) \sqrt{\tilde{V}(\tilde{e}(0))}+k
$$

where $k=\frac{4}{3 a} K_{M} \bar{\theta}^{-\sigma_{1}^{k}}\left(\mu(S)+\frac{1}{\lambda_{m}(S)}\right) B_{\tilde{y}}$. As a result, $\tilde{V}(\tilde{e}(t))$ is bounded with an ultimate bound equals to $k$ and so is $\tilde{e}(t)$, or equivalently $e(t)$. In the sequel, one shall denote $B_{e}$ the upper bound of $\|e(t)\|$, i.e.

$$
\forall t \geq 0:\|e(t)\| \leq B_{e}
$$

To end the proof, one shall firstly show that $\tilde{y}(t)$ converges to zero and then the convergence to zero of $e(t)$ shall be proven. Let us show that $\|\tilde{y}(t)\|$ converges to zero. In fact, one shall show not only that $\tilde{y}$ converges to zero, but also it does it exponentially. This can be done by a contradiction argument. Indeed, suppose that $\tilde{y}$ does not exponentially converge to zero. This implies that: $\forall c>0 ; \forall \alpha>0 ; \forall T^{\star}>T ; \exists t^{\star}>T^{\star}:\left\|\tilde{y}\left(t^{\star}\right)\right\|>c e^{-\alpha t^{\star}}$. But since the function $t \mapsto\|\tilde{y}(t)\|-c e^{-\alpha t}$ is continuous, one also has:

$$
\begin{gathered}
\forall c>0 ; \forall \alpha>0 ; \forall T^{\star}>T ; \exists T_{1}>0 ; \forall t \in\left[t^{\star}, t^{\star}+T_{1}\right]: \\
\|\tilde{y}(t)\|>c e^{-\alpha t} \geq c e^{-\alpha\left(t^{\star}+T_{1}\right)}
\end{gathered}
$$

Now, since $\|\tilde{y}\|$ is bounded and by choosing $\rho>B_{\tilde{y}}^{2}$, one has for $t \geq t^{\star}:$

$$
g(t) \geq \frac{M}{1+B_{\tilde{y}}^{2}}
$$

Now, choose $\mathrm{M}$ as follows

$$
M=\frac{\lambda\left(1+B_{\tilde{y}}^{2}\right)}{\gamma_{m}}
$$


where $\gamma_{m}=\inf \left\{\gamma(\|\tilde{y}(t)\|) ; t \in\left[t^{\star}, t^{\star}+T_{1}\right]\right\}$ and $\lambda$ is a positive constant satisfying $\lambda>\theta_{c}^{\delta_{1}+\frac{1}{2}} \frac{\mu_{2}}{\mu_{1}}$. Using (51), inequality (36) becomes $\dot{V}(t) \leq-\frac{1}{4} a(\theta+1) V(t)+\frac{\theta_{c}^{\delta_{1}+\frac{1}{2}}}{4} V-\frac{\mu_{1}}{4 \mu_{2}} \frac{M}{\left(1+B_{\tilde{y}}^{2}\right)} \gamma_{m} V$. Substituting $M$ by its expression, (52), in (36), one obtains for all $t \in\left[t^{\star}, t^{\star}+T_{1}\right]:$

$$
\dot{V} \leq-\left(\frac{\mu_{1}}{\mu_{2}} \lambda-\theta_{c}^{\delta_{1}+\frac{1}{2}}\right) \frac{1}{4} V
$$

Integrating (53) on $\left[t^{\star}, t^{\star}+T_{1}\right]$, one gets: $V\left(\bar{e}\left(t^{\star}+T_{1}\right)\right) \leq$ $\exp \left(-\frac{T_{1}}{4}\left(\frac{\mu_{1}}{\mu_{2}} \lambda-\theta_{c}^{\delta_{1}+\frac{1}{2}}\right)\right) V\left(\bar{e}\left(t^{\star}\right)\right)$, or equivalently: $\left\|\bar{e}\left(t^{\star}+T_{1}\right)\right\| \leq$ $\sqrt{\mu(S)} \exp \left(-\frac{T_{1}}{4}\left(\frac{\mu_{1}}{\mu_{2}} \lambda-\theta_{c}^{\delta_{1}+\frac{1}{2}}\right)\right)\left\|\bar{e}\left(t^{\star}\right)\right\|$. Now, according to $(27)$ and (14) and using (13), one has for $\theta(t) \geq 1$ :

$$
\left\|\bar{e}^{k}(t)\right\| \leq\left\|e^{k}\right\| \leq \theta^{\sigma_{1}^{k}+\lambda_{k} \delta_{k}}\left\|\bar{e}^{k}\right\|=\theta^{\lambda_{1} \delta_{1}}\left\|\bar{e}^{k}\right\|
$$

As a result, one has

$$
\left\|\tilde{y}\left(t^{\star}+T_{1}\right)\right\| \leq\left\|e\left(t^{\star}+T_{1}\right)\right\| \leq \theta_{\max }^{\lambda_{1} \delta_{1}}\left\|\bar{e}\left(t^{\star}+T_{1}\right)\right\|
$$

Combining (54) and (55), one gets $\left\|\tilde{y}\left(t^{\star}+T_{1}\right)\right\| \leq \sqrt{\mu(S)} \theta_{\max }^{\lambda_{1} \delta_{1}}$

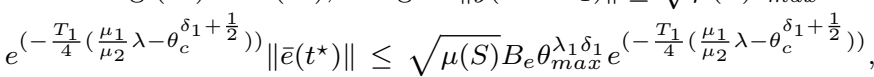
where $B_{e}$ is the upper bound of $e(t)$ as given by (49). Now, it is clear that one can choose $\lambda$ high enough such that:

$$
\sqrt{\mu(S)} B_{e} \theta_{\max }^{\lambda_{1} \delta_{1}} e^{\left(-\frac{T_{1}}{4}\left(\frac{\mu_{1}}{\mu_{2}} \lambda-\theta_{c}^{\delta_{1}+\frac{1}{2}}\right)\right)}<\frac{1}{2} c e^{-\alpha\left(T_{1}+t^{\star}\right)}
$$

Combining (50) and (56) leads to a contradiction, i.e. $0<$ $c e^{-\alpha\left(T_{1}+t^{\star}\right)}<\left\|\tilde{y}\left(t^{\star}+T_{1}\right)\right\|<\frac{1}{2} c e^{-\alpha\left(T_{1}+t^{\star}\right)}$. The convergence to zero of $\tilde{y}$ is proven and thus one has:

$$
\exists c>0 ; \alpha>0 ; \exists T^{\star}>T ; \forall t>T^{\star}:\|\tilde{y}(t)\| \leq c e^{-\alpha t}
$$

To end the proof of the Theorem, one has to prove the exponential convergence to zero of $\|e(t)\|$. Let us proceed as above when proving the boundedness of $e(t)$ and let us consider again the same change of variable given by (43). Proceeding as above and miming (48), one gets for $t \geq T^{\star}$ where $T^{\star}$ is given by (57):

$$
\sqrt{\tilde{V}(\tilde{e}(t))} \leq \exp \left(-\frac{3}{8} a \bar{\theta} t\right) \sqrt{\tilde{V}(\tilde{e}(0))}+m c e^{-\alpha t}
$$

where $\bar{\theta}$ is given by (44) and $m=\frac{4}{3 a} K_{M} \bar{\theta}^{-\sigma_{1}^{k}}\left(\mu(S)+\frac{1}{\lambda_{m}(S)}\right)$. It is clear from (58) that $\tilde{V}(\tilde{e}(t))$ exponentially converges to zero. This ends the proof of the theorem.

\subsection{Tuning mechanism design}

The equations of the observer involve three design parameters $M$ $T$ and $\rho$. The choice of the parameter $\rho$ is not crucial. It is mainly introduced to saturate the integral term $\frac{1}{T} \int_{\max (0, t-T)}^{t}\|\tilde{y}(\tau)\|^{2} d \tau$. As a result, a very high value generally does the job in practice. Indeed, this parameter was never considered in all the simulation we have carried out. We now propose to discuss the choice of the other design parameters of the observer, namely the function $\gamma$ and the parameters $M$ and $T$.

Choice of $\gamma$ : It is required for the real-valued function $\gamma(\cdot)$ to be non negative, non decreasing and bounded with $\gamma(0)=0$. Of course many real functions satisfy such requirements. We give here two expressions that have been used in the examples given in simulation. - Choice 1: $\gamma(\xi)=\frac{\xi^{2}}{1+\xi^{2}}$ where $\xi \in R_{+}^{\star}$

- Choice 2: $\gamma(\xi)=\tanh \xi^{2}$ where $\xi \in R_{+}^{\star}$.

Tuning of $M$ and $T$ : For clarity purposes, let us rewrite $g(t)$ as follows: $g(t)=\frac{M}{1+\min \left(\rho, \mathcal{P}_{T}(t)\right)}$, where $\mathcal{P}_{T}(t)=\frac{1}{T} \int_{\max (0, t-T)}^{t}\|\tilde{y}(\tau)\|^{2} d \tau$ and it represents the power of the output observation error, $\tilde{y}(t)$, calculated on a moving window with a width equal to $T$. It is clear that the function $g(t)$ is bounded as follows: $\forall t \geq 0: \frac{M}{1+\rho} \leq g(t) \leq M$.
The parameter $M$ can be taken very high in the absence of noise measurements. Such high values allows to the parameter $\theta(t)$ to reach also high values which quickly leads to the vanishing of the observation error. The values of the parameter $\theta(t)$ decreases then to the predefined value 1 . However, the adoption of high values of $M$ are to be avoided as far as possible in practice due to the unavoidable presence of noise measurements. Indeed, as said above, high values of $M$ make $\theta(t)$ high and the observer becomes very sensitive to the noise. In order to take into account the presence of the noise measurements, the value of $M$ is divided by the power of the output observation error calculated on a moving window with a width equal to $T$. It is worth noticing that small values of $T$ are advised in the presence of noise measurements with a variance that varies significantly and relatively quickly in a continuous manner. On the contrary, relatively high values of $T$ have to adopted where the variance of the noise measurements is constant or varies slowly.

\section{EXAMPLE}

In order to illustrate the performances of the dynamic high gain observer, one considers the following nonlinear system:

$$
\left\{\begin{array}{l}
\dot{x}_{1}=x_{2}+x_{2}^{3}+u_{1}-x_{1} \\
\dot{x}_{2}=10 \cos \left(10 x_{2}\right)+5 \cos (t) \\
\dot{x}_{3}=\left(a-u_{2}\right) x_{5}-u_{2} x_{6}-x_{3} \\
\dot{x}_{4}=u_{2} x_{5}+u_{2} x_{6}-x_{4}+x_{2} \\
\dot{x}_{5}=-x_{5}-x_{8}^{3}-\arctan \left(x_{8}\right)-\sin \left(x_{7}\right) \\
\dot{x}_{6}=x_{7}^{3}+x_{7}+\frac{x_{5}}{1+x_{5}^{2}}-x_{6} \\
\dot{x}_{7}=x_{8}^{3}+\tanh \left(x_{8}\right)+x_{5}^{2}-x_{7} \sin \left(x_{7}\right) \\
\dot{x}_{8}=-x_{8}-20 \cos \left(x_{5} x_{2}\right) \\
y=\left(\begin{array}{lll}
x_{1} x_{3} x_{4} x_{6}
\end{array}\right)^{\mathrm{T}}
\end{array}\right.
$$

One can check that the following map $\Phi: R^{8} \longrightarrow R^{9}, x \mapsto z=\Phi(x)$ with $z_{1}=x_{1}, z_{2}=x_{2}+x_{2}^{3}, z_{3}=x_{3}, z_{4}=x_{4}, z_{5}=\left(a-u_{2}\right) x_{5}$, $z_{6}=u_{2} x_{5}, z_{7}=x_{6}, z_{8}=x_{7}^{3}+x_{7}$ and $z_{9}=\left(1+3 x_{7}^{2}\right)\left(x_{8}^{3}+\tanh x_{8}\right)$ puts system (59) under form (1) with

- $x^{1}=\left(\begin{array}{ll}x_{1}^{1} & x_{2}^{1}\end{array}\right)^{\mathrm{T}}$ with $x_{1}^{1}=z_{1}, x_{2}^{1}=z_{2}$.

- $x^{2}=\left(\begin{array}{ll}x_{1}^{2} & x_{2}^{2}\end{array}\right)^{\mathrm{T}}$ with $x_{1}^{2}=\left(\begin{array}{ll}z_{3} & z_{4}\end{array}\right)^{\mathrm{T}} ; x_{2}^{2}=\left(\begin{array}{ll}z_{5} & z_{6}\end{array}\right)^{\mathrm{T}}$.

- $x^{3}=\left(\begin{array}{lll}x_{1}^{3} & x_{2}^{3} & x_{3}^{3}\end{array}\right)^{\mathrm{T}}$ with $x_{1}^{3}=z_{7}, x_{2}^{3}=z_{8}$ and $x_{3}^{3}=z_{9}$.

The output $y$ is then partitioned as $y=\left(\begin{array}{lll}y_{1} & y_{2} & y_{3}\end{array}\right)^{\mathrm{T}}$ with $y_{1}=$ $z_{1} ; y_{2}=\left(\begin{array}{ll}z_{3} & z_{4}\end{array}\right)^{\mathrm{T}}$ and $y_{3}=z_{7}$. One has $\lambda_{1}=\lambda_{2}=2$ and $\lambda_{3}=3$. An observer of the form (20) is synthesized for system (59) and one has $\delta_{1}=8 ; \delta_{2}=4$. Due to the lack of space, we don't give the equations of the observer. In what follows, one shall give simulation results obtained with the following initial conditions: $x_{1}(0)=1, x_{2}(0)=0.8$ and $x_{i}(0)=1, i=3, \ldots, 8, \hat{x}_{i}(0)=1, i=$ $\{1,3,4,6\} ; \hat{x}_{j}(0)=-1, j=\{5,7,8\} ; \hat{x}_{2}(0)=2$ and the inputs $u_{1}=$ $2 \sin \left(\frac{t}{\pi}\right), u_{2}=\cos (2 \pi t)$. In order to simulate practical situations and to show the insensitivity with respect to noise measurements of the dynamic high gain observer, all the outputs of system (59) has been corrupted by a noise measurements with zero mean value and a standard deviation equals to $\sqrt{0.01}$. The values of the parameters $M, T, a$ and $\sigma$ used in simulation were respectively equal to 50,1 , 10 and 1 . Concerning the function $\gamma$, it was specified as follows in this application: $\gamma(\tilde{y})=\frac{\|\tilde{y}\|^{2}}{1+\|\tilde{y}\|^{2}}$. The estimation of all missing state variables provided by the observer is given in figure (1) which clearly highlight its performance. The evolution of the design parameter $\theta(t)$ is given in figure (2). Such figure shows that the parameter $\theta$ has decreased to low values as soon as the state estimates coincided with their real unknown values. At these lows values, the observer still provide accurate estimate while keeping a good behavior with respect to noise measurements. For comparison purposes, one has simulated the proposed observer using two different constant values, 5 and 10 for the design parameter $\theta$. The corresponding results are given in figure (3). Notice that the dynamic high gain observer outperforms the usual high gain observer from the point of view of sensitivity to noise measurements. 

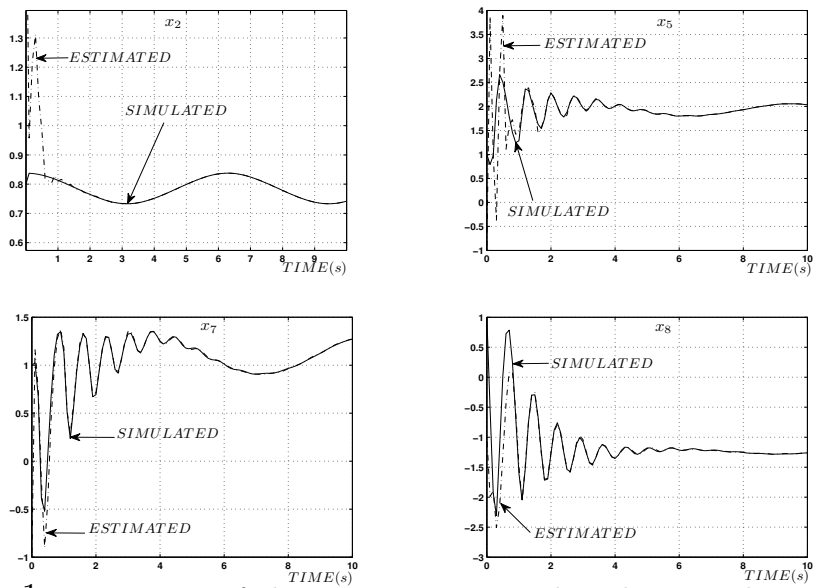

Fig. 1. Estimation of the missing states with a dynamic design parameter.

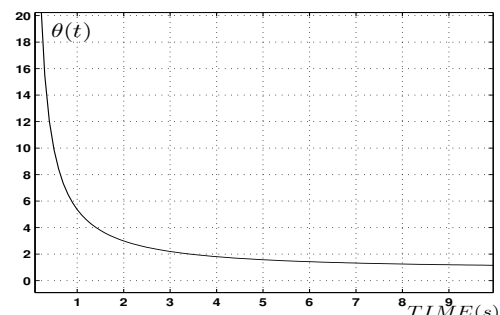

Fig. 2. Evolution of $\theta(t)$.
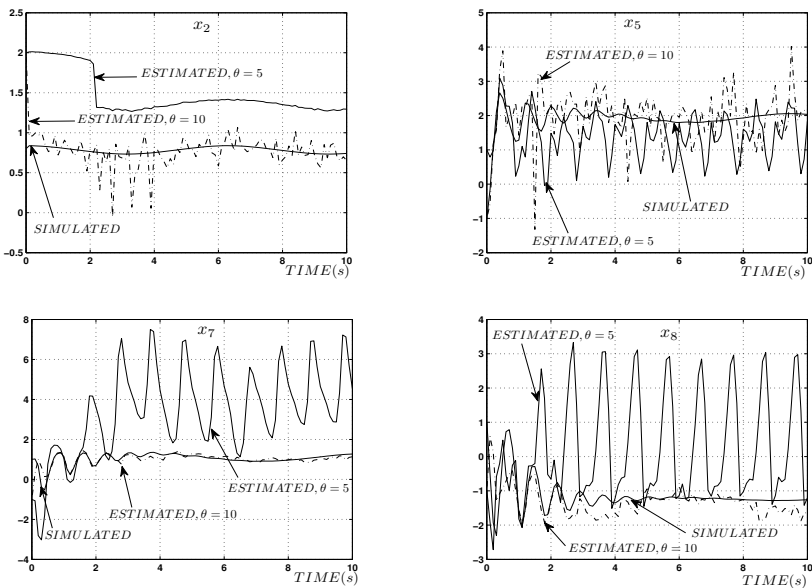

Fig. 3. Estimation of the missing states with different values of the constant design parameter.

\section{CONCLUSION}

A dynamic high gain observer for a most general class of MIMO nonlinear systems that are observable for any inputs is proposed. The main contribution consists in the novelty of the observer whose gain is coming from a Riccatti equation. Simulation results demonstrated the well behavior of the proposed high gain observer with respect to the unavoidable noise measurements.

\section{REFERENCES}

Arcak, M. and Kokotović, P. (2001). Nonlinear observers: a circle criterion design and robustness analysis. Automatica, 37, 19231930.

Busawon, K., Farza, M., and Hammouri, H. (1998). Observer design for a special class of nonlinear systems. International Journal of Control, 71, 405-418.

Cho, H. and Ro, K. (2005). LMI-based sliding-mode observer design method. Control, Theory and Applications, IEE Proceedings, 152, 113-115.
Fan, X. and Arcak, M. (2003). Observer design for systems with multivariable monotone nonlinearities. Systems \& Control letters, $50,319-330$

Farza, M., Hammouri, H., Jallut, C., and Lito, J. (1999). State observation of a nonlinear system : Application to (bio)chemical processes. AIChE Journal, 45, 93-106.

Farza, M., M'Saad, M., and Rossignol, L. (2004). Observer design for a class of MIMO nonlinear systems. Automatica, 40, 135-143.

Gauthier, J., H., H.H., and Othman, S. (1992). A simple observer for nonlinear systems - application to bioreactors. IEEE Trans. on Aut. Control, 37, 875-880.

Guay, M. (2002). Observer linearization by output-dependent timescale transformations. IEEE Transactions on Automatic Control, 47, 1730-1735.

Hammouri, H. and Farza, M. (2003). Nonlinear observers for locally uniformly observable systems. ESAIM J. on Control, Optimisation and Calculus of Variations, 9, 353-370.

Hou, M. and Pugh, A.C. (1999). Observer with linear error dynamics for nonlinear multi-output systems. Syst. Contr. Lett., 37, 1-9.

Kalman, R. and Bucy, R. (1961). New results in linear filtering and prediction theory. J. Basic Eng., 95-108.

Khalil, H. and Saberi, A. (1987). Adaptive stabilization of a class of nonlinear systems using high gain feedback. IEEE Transactions on Automatic Control, 11, 1031-1035.

Krener, A. and Isidori, A. (1983). Linearization by output injection and nonlinear observers. Syst. Contr. Lett., 3, 47-52.

Krishnamurthy, P. and Khorrami., F. (2002). Generalized adaptive output-feedback form with unknown parameters multiplying high output relative-degree states. In Proceedings of the 41 st IEEE Conference on Decision and Control.

Krishnamurthy, P. and Khorrami, F. (2004). Dynamic high-gain scaling: State and output feedback with application to systems with ISS appended dynamics driven by all states. IEEE Transactions on Automatic Control, 49(12), 2219-2237.

Lei, H. and Lin, W. (2006). Universal adaptive control of nonlinear systems with unknown growth rate by output feedback. Automatica, 42, 1783-1789.

Liu, F., Farza, M., M'Saad, M., and Hammouri, H. (2008). Observer design for a class of uniformly observable MIMO nonlinear systems with coupled structure. In Proceedings of the 17th IFAC World Congress.

Praly, L. (2003). Asymptotic stabilization via output feedback for lower triangular systems with output dependent incremental rate. IEEE Transactions on Automatic Control, 48(6), 1103-1108.

Rajamani, R. (1998). Observers for Lipschitz Nonlinear Systems. IEEE Transactions on Automatic Control, 43(3), 397-401.

Scharf, L. and Sigurdsson, S. (1984). Fixed point implementation of fast kalman predictors. IEEE Trans. on Automat. Contr., 9, 850-852.

Shen, Y., Shen, W., Jiang, M., and Huang, Y. (2010). Semiglobal finite-time observers for multi-output nonlinear systems. International Journal of Robust and Nonlinear Control, 20, 789801.

Shen, Y. and Xia., X. (2008). Semi-global finite-time observers for nonlinear systems. Automatica, 44, 3152-3156.

Shim, H., Son, Y., and Seo, J. (2001). Semi-global observer for multioutput nonlinear systems. Syst. Contr. Lett., 42, 233-244.

Souleiman, I., Glumineau, A., and Schreirer, G. (2003). Direct transformation of nonlinear systems into state affine MISO form and nonlinear observers design. IEEE Trans. on Automat. Contr., 48, 2191-2196.

Tan, C. and Edwards, C. (2001). An LMI approach for designing sliding mode observers. International Journal of Control, 74, 1559-1568.

Xia, X. and Gao, W. (1989). Nonlinear observer design by observer error linearization. SIAM J.Control Optim., 27(1), 199-216. 